\title{
A THREE-DIMENSIONAL AEROSOL VARIATIONAL DATA ASSIMILATION SYSTEM FOR AIRCRAFT AND SURFACE OBSERVATIONS
}

\author{
Zengliang Zang*, Xiaobin Pan, Wei You, Yanfei Liang \\ Institute of Meteorology and Oceanography, National University of defense technology, Nanjing 211101, China
}

Commission VI, WG III/8

KEY WORDS: Data assimilation, WRF/Chem, Aerosol, Aircraft

\begin{abstract}
:
A three-dimensional variational data assimilation system is implemented within the Weather Research and Forecasting/Chemistry model, and the control variables consist of eight species of the Model for Simulation Aerosol Interactions and Chemistry scheme. In the experiments, the three-dimensional profiles of aircraft speciated observations and surface concentration observations acquired during the California Research at the Nexus of Air Quality and Climate Change field campaign are assimilated. The data assimilation experiments are performed at 02:00 local time 2 June 2010, assimilating surface observations at 02:00 and aircraft observations from 01:30 to 02:30 local time. The results show that the assimilation of both aircraft and surface observations improves the subsequent forecasts. The improved forecast skill resulting from the assimilation of the aircraft profiles persists a time longer than the assimilation of the surface observations, which suggests the necessity of vertical profile observations for extending aerosol forecasting time.
\end{abstract}

\section{INTRODUCTION}

Data assimilation (DA) has been increasingly used to improve aerosol analysis and prediction in recent years in association with atmospheric chemical models(Benedetti et al., 2009a, Zhang et al., 2008a; Mangold et al, 2011a). Data assimilation aims to integrate all available observations into a model to produce optimal estimates of aerosol fields, then used to initialize the model to improve the subsequent forecast. For an atmospheric chemical model, the improved forecast skill usually sharply declines within a few hours after the forecasting model is initialized using data assimilation analysis, although a limited positive impact of data assimilation on forecasts may persist beyond $24 \mathrm{~h}$. The quick loss of the improved forecast skill may be related to uncertainties in surface fluxes, dynamic properties of aerosol variability, model performance, and data assimilation performance. The inadequacy of aerosol data assimilation performance continues to be a challenge in aerosol data assimilation. This is primarily due to the high cost of measurements and the large number of aerosol state variables. A sophisticated aerosol model may explicitly treat more than a dozen species, and each species involves different size bins, which increase the number of state variables in multiples. A large number of state variables require a large number of observations in data assimilation. The last decade has witnessed great progress in the technology of aerosol measurement, leading to the establishment of a variety of observation networks (Diner et al., 2004a). However, the measurements are limited to the total concentration of surface PM2.5 or PM10(Weber et al., 2003a). Satellite measurements provide global coverage, but the most common observations are aerosol optical depths (AODs) and are often unavailable due to clouds and high surface reflectance(van Donkelaar et al., 2010; Levy et al., 2010a; Lee et al., 2011a). A global lidar network has been established to measure aerosol scattering profiles but is limited to merely tens of stations(Wang et al., 2013a; 2014a). The available observations are far inadequate to constrain all the variables in a high-resolution model using data assimilation. Thus, it is necessary to develop a data assimilation system that can assimilate different kind of observation to produce an optimal aerosol analysis fields.

In this paper, we examine the relative impact of vertical profiles and surface observations on forecasts during the California Research at the Nexus of Air Quality and Climate Change (CalNex) field campaign. The CalNex 2010 campaign was a major climate and air quality study in California conducted by the National Oceanic and Atmospheric Administration (NOAA) and the California Air Resources Board (ARB) (http://www.esrl.noaa.gov/csd/projects/calnex). During the experiments, surface observations were acquired from an enhanced network and vertical profiles from a few flights made by an aircraft operated by NOAA. We use a three-dimensional variational data assimilation (3DVAR) system ( $\mathrm{Li}$ et al., 2013a) to assimilate both surface observations and aircraft vertical profile measurements. An Observing System Experiment (OSE) is conducted to characterize their impact on analysis and forecast.

\section{OBSERVATIONS}

The CalNex field campaign took place from May to July 2010 and was a multi-institution effort to address both air quality and climate change in the Los Angeles Basin and the San Joaquin Valley. During the CalNex field campaign, NOAA conducted

\footnotetext{
* Zengliang Zang, zzlqxxy@163.com

This research was supported by National Science Foundation of China(41775123)
} 
several aircraft flights to acquire sophisticated airborne gas and particulate three-dimensional (3D) profile measurements.

In the experiments presented later, two types of observations are assimilated. One consists of the surface total mass concentration of $\mathrm{PM}_{2.5}$, and the other of speciated aerosol mass concentration $3 \mathrm{D}$ profiles from aircraft fights.

Hourly surface $\mathrm{PM}_{2.5}$ concentrations are obtained from ARB. There are 42 surface measurement sites in Southern California (Fig 1). The speciated vertical profiles were assimilated from one flight made on June 2, 2010. The aircraft took off at about 01:00 local time (Pacific Daylight Time, PDT) and landed at about 07:00 PDT, corresponding universal time is from 08:00 to 14:00 UTC around Los Angeles Basin (Fig 1).

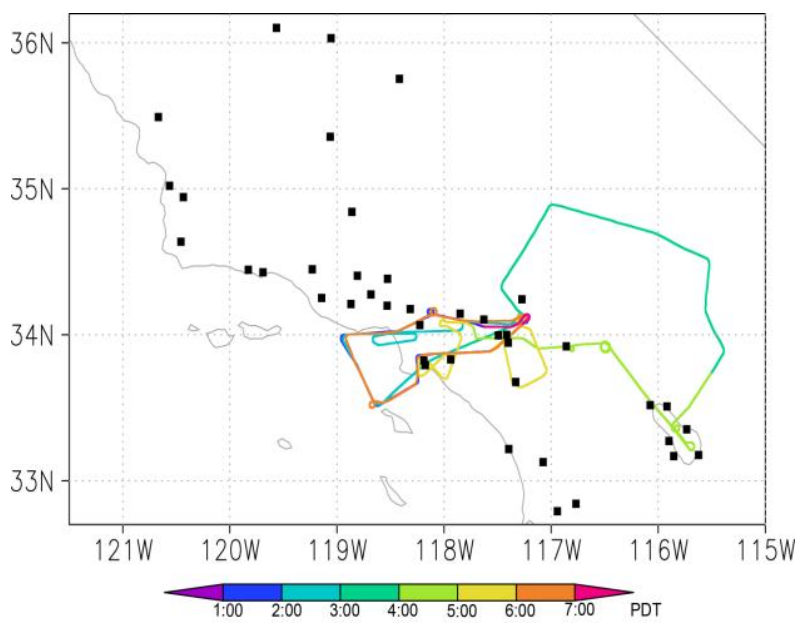

Figure 1 Map of the surface sites (black squares) and aircraft flight track on June 2, 2010 (colored curve)

\section{MODEL CONFIGURATION AND DATA ASSIMILATION ALGORITHM}

We used WRF/Chem (v3.5) to simultaneously predict weather and atmospheric (chemistry) composition. The WRF/Chem model configuration has a triple-nested domain, with a resolution of $36 \mathrm{~km}, 12 \mathrm{~km}, 4 \mathrm{~km}$, respectively (Fig 2). The MOSAIC (Model for Simulating Aerosol Interactions and Chemistry) scheme (Zaveri et al., 2008) is selected for the representation of aerosol processes.

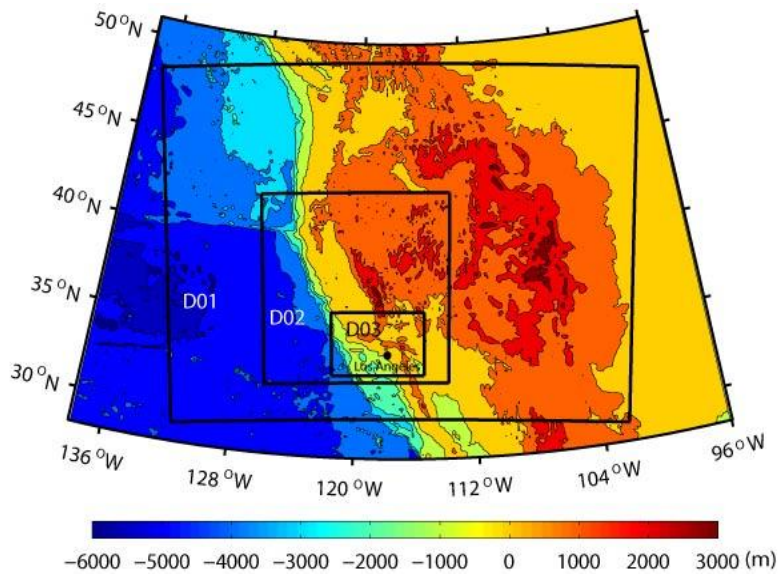

The 3DVAR used here allows combination of size bins and species to form so called lumped variables. Li et al. (2013) reduced the number of the control variables to five. Here we combine the size bins for each species in the MOSAIC scheme

(Fast et al., 2006a). The total aerosol concentrations of the eight species are the control variables in this 3DVAR system. Since the three new control variables are $\mathrm{Cl}, \mathrm{Na}$ and $\mathrm{NH}_{4}$ concentrations, the aircraft measurements of $\mathrm{NH}_{4}$ concentrations can then be directly assimilated.

\section{DATA ASSIMILATION AND FORECASTING EXPERIMENTS}

Four experiments are conducted to characterize the impact of the surface and aircraft measurements on 3DVAR analyses and PM2.5 forecasts. The first is a control experiment, which is a simulation driven by the forecast of the meteorological field without aerosol DA, often known as a free run and denoted as Control; the second a DA experiment that assimilates only the surface PM2.5 measurements, denoted as DA-sfc; the third a DA experiment that assimilates only the aircraft profile measurements, denoted as DA-air, and the fourth a DA experiment that assimilates the surface and aircraft measurements simultaneously, denoted as DA-both. Such experiments are often referred to as OSE.

Using the model configurations described in Sec 3.1, all experiments began from 02:00 PDT 2 June 2010, and then ran for 39 hours, ending at 17:00 PDT 3 June 2010. The DA experiments are applied only in the innermost domain (Fig 3) of the model.

\section{RESULTS AND ANALYSES}

To characterize the data assimilation analyses, we compare the results of the four experiments against the observations that are assimilated, which is known as "sanity check." Three basic statistical measures, mean bias (BIAS), root-mean-spuare error (RMSE), and correlation coefficient (CORR), are utilized for the evaluations.

Figure 3 shows the scatter plots of the model versus the observed surface $\mathrm{PM}_{2.5}$ mass concentrations at 02:00 PDT, 2 June 2010, the time when the model was initialized. Compared with the Control experiment, all the three DA experiments are closer to the observed $\mathrm{PM}_{2.5}$. The result indicates that the aircraft observations are accordant with the surface observations, though they are not in same levels. Also, the improvement of DA-air is mainly around the area of Los Angeles, there are little influence on the other areas far away the area of aircraft observations. The bias of the DA-both experiment is the smallest among all the experiments, while the RMSE and CORR are comparable with that of the DA-sfc.
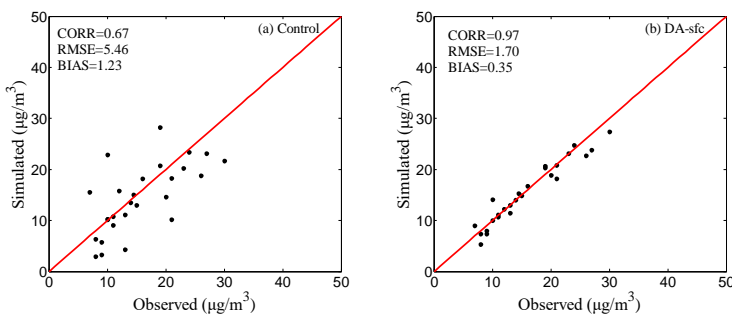

Figure 2 Three-nested model domains. The black square indicates the location of Los Angeles. Colors indicate topographic elevations. 

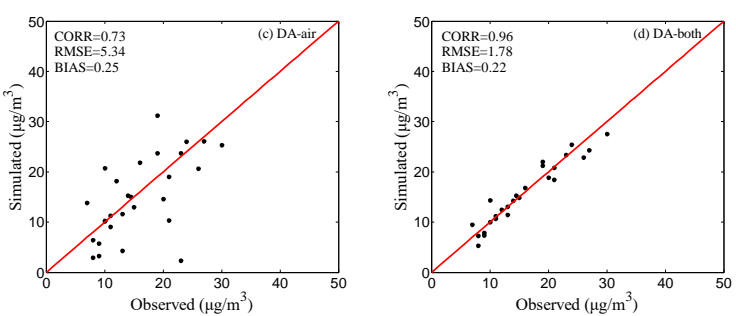

Figure 3 Scatter plots of observed concentrations of $\mathrm{PM}_{2.5}$ versus simulated concentrations of $\mathrm{PM}_{2.5}$ from the experiments of Control (a), DA-sfc (b), DA-air (c) and DA-both (d) surface over 02:00 PDT initializations.

Figure 4 shows the CORR, RMSE and BIAS of the $\mathrm{PM}_{2.5}$ forecasts against the surface observations as a function of forecasting duration. The correlations of the three DA forecasts are higher than that of the Control forecast during the earlier forecasts. The CORR of the DA-both is very similar with that of the DA-sfc during the first four hours. For the forecast between 4 and 18 hours, the CORR is persistently higher with DA-both than with DA-sfc. The RMSE and BIAS of the DA-both experiment is persistently lower during the first 15 hours than that of the other experiments. Interestingly, the first three hour forecast is more skillful with DA-sfc than DA-air in terms of both CORR and RMSE. Between 4 and 15 hours, the forecast is more skillful with DA-air than DA-sfc. It suggests that the assimilation of aircraft observations can result in positive and more persistent effect on surface $\mathrm{PM}_{2.5}$ forecast.
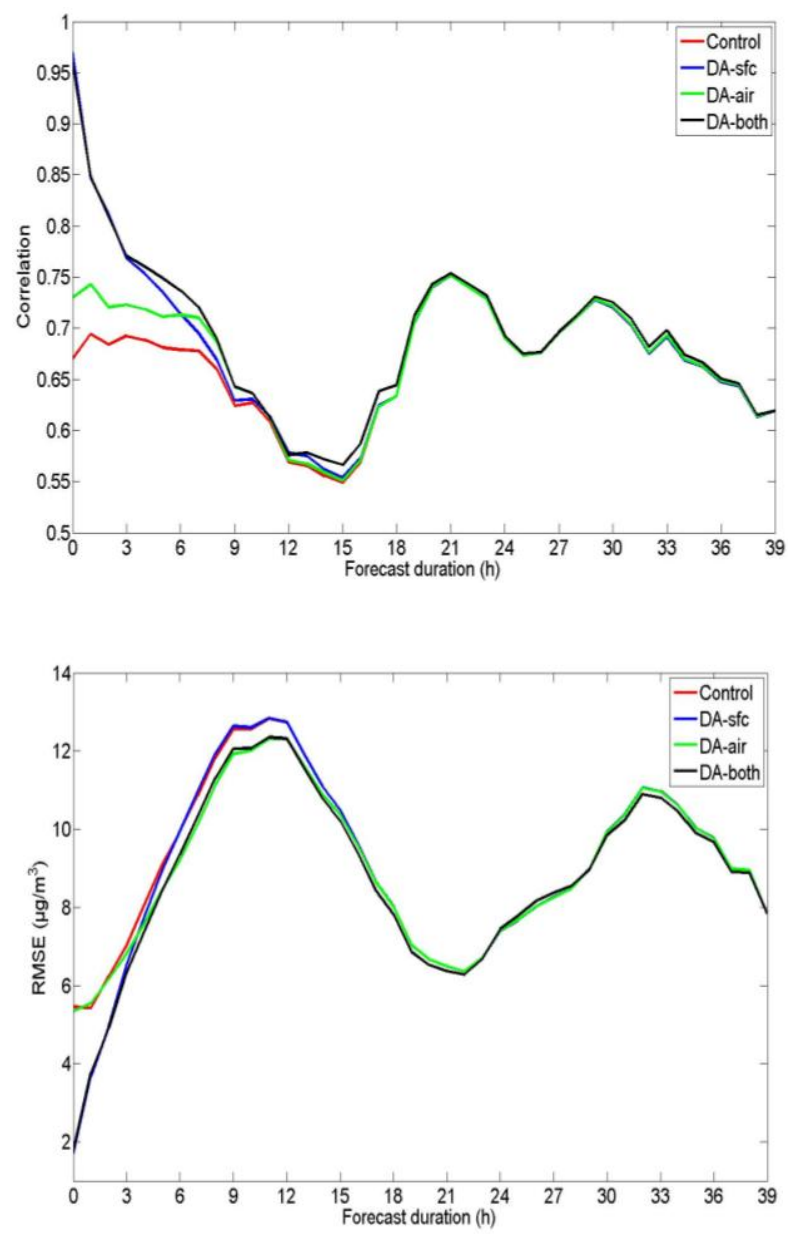

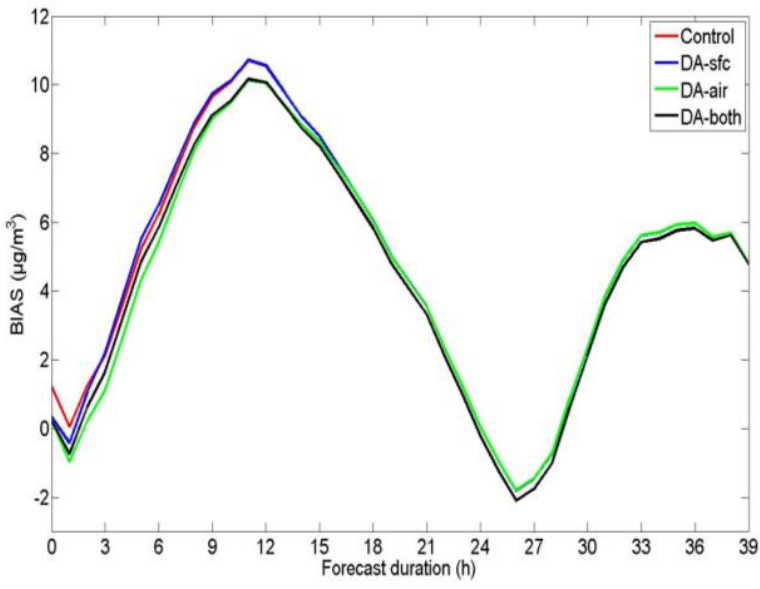

Fig 4 Correlations (a), root-mean-square errors (RMSE) (b) and BIAS (c) of the total $\mathrm{PM}_{2.5}$ concentration forecasts against observations as a function of forecast duration.

\section{SUMMARY AND CONCLUSIONS}

The results from the experiments suggest that the aircraft vertical profiles observations, although localized within a limited area, contribute more to forecast skill improvement, supporting a hypothesis that vertical profiles are more effective in extending forecast skills. Such results might be the consequence of ineffectiveness of the propagation of the information of surface measurements from the surface to the upper air in the DA scheme.

We note that, due to the short time of availability of vertical profile measurements, the results are based on DA experiments performed at one single time. This is thus a case study. The results are more suggestive than conclusive. An extended period of time or experiments with other field campaigns are needed to confirm the results presented here.

\section{ACKNOWLEDGEMENTS}

This research was supported by the National Natural Science Foundation of China (41275128).

\section{REFERENCES}

Benedetti, A., Morcrette, J.-J., Boucher, O., Dethof, A., Engelen, R. J. and co-authors. 2009. Aerosol analysis and forecast in the European Centre for Medium-Range Weather Forecasts Integrated Forecast System: 2. Data assimilation. J. Geophys. Res. 114, D13205.

Diner, D. J., Ackerman, T. P., Anderson, T. L., Bosenberg, J., Braverman, A. J. and co-authors. 2004. PARAGON: An integrated approach for characterising aerosol climate impacts and environmental interactions. B. Am. Meteorol. Soc. 85, 1491-1501.

Fast, J. D., Gustafson Jr, W. I., Easter, R. C., Zaveri, R. A., Barnard, J. C. and co-authors. 2006. Evolution of ozone, particulates, and aerosol direct radiative forcing in the vicinity of Houston using a fully coupled meteorology-chemistryaerosol model. J. Geophys. Res. 111, D21305.

Lee, H. J., Liu, Y., Coull, B. A., Schwartz, J. and Koutrakis, P. 2011. A novel calibration approach of MODIS AOD data to 
predict $\mathrm{PM}_{2.5}$ concentrations. Atmos. Chem. Phys. 11, 79918002.

Levy, R. C., Remer, L. A., Kleidman, R. G., Mattoo, S., Ichoku, C. and co-authors. 2010. Global evaluation of the Collection 5 MODIS dark-target aerosol products over land. Atmos. Chem. Phys. 10, 10399-10420.

Li, Z., Zang, Z., Li, Q. B., Chao, Y., Chen, D. and co-authors. 2013. A three-dimensional variational data assimilation system for multiple aerosol species with WRF/Chem and an application to $\mathrm{PM}_{2.5}$ prediction. Atmos. Chem. Phys. 13, 4265-4278.

Mangold, A., De Backer, H., De Paepe, B., Dewitte, S., Chiapello, I. and co-authors. 2011. Aerosol analysis and forecast in the European Centre for Medium-Range Weather Forecasts Integrated Forecast System: 3. Evaluation by means of case studies. J. Geophys. Res. 116, D03302.

Wang, Y., Sartelet, K. N., Bocquet, M. and Chazette, P. 2013. Assimilation of ground versus lidar observations for $\mathrm{PM}_{10}$ forecasting. Atmos. Chem. Phys. 13, 269-283.

Wang, Y., Sartelet, K. N., Bocquet, M. and Chazette, P. 2014. Modelling and assimilation of lidar signals over Greater Paris during the MEGAPOLI summer campaign. Atmos. Chem. Phys. 14, 3511-3532.

van Donkelaar, A., Martin, R. V., Brauer, M., Kahn, R., Levy, R. and co-authors. 2010. Global estimates of ambient fine particulate matter concentrations from satellite-based aerosol optical depth: Development and application. Environ. Health Persp. 118, 847-855.

Weber, R., Orsini, D., Duan, Y., Baumann, K., Kiang, C. S. and co-authors. 2003. Intercomparison of near real time monitors of $\mathrm{PM}_{2.5}$ nitrate and sulfate at the U.S. Environmental Protection Agency Atlanta Supersite. J. Geophys. Res. 108(D7), 84218435 .

Zaveri, R. A., Easter, R. C., Fast, J. D. and Peters. L. K. 2008. Model for Simulating Aerosol Interactions and Chemistry (MOSAIC). J. Geophys. Res. 113, D13204.

Zhang, J., Reid, J. S., Westphal, D., Baker, N. and Hyer, E. 2008. A System for Operational Aerosol Optical Depth Data Assimilation over Global Oceans. J. Geophys. Res. 113, D10208. 